\title{
Comparison of Rhyming and Word Generation with FMRI
}

\author{
Joseph T. Lurito, '* David A. Kareken, ${ }^{2}$ Mark J. Lowe, ' \\ Shen Hsing A. Chen, ${ }^{2}$ and Vincent P. Mathews'
}

${ }^{1}$ Department of Radiology, Indiana University School of Medicine, Indianapolis, Indiana

${ }^{2}$ Department of Neurology, Indiana University School of Medicine, Indianapolis, Indiana

\begin{abstract}
Functional magnetic resonance imaging (FMRI) has been successfully used to non-invasively map language function, but has several disadvantages. These include severe motion sensitivity, which limits overt verbal responses in behavioral paradigms, such as word generation. The lack of overt responses prevents behavioral validation, making data interpretation difficult. Our objective was to compare the FMRI activation patterns of a novel silent rhyme determination task requiring a non-verbal response, to covert word generation from visually presented letters. Five strongly right-handed subjects performed both tasks during multi-slice coronal echo-planar T2*-weighted FMRI. Single subject activation maps were generated for each task by correlation analysis of single pixel time series to a boxcar reference function. These maps for the two tasks were separately interpolated to $256^{3}$, transformed into Talairach space, summed, and thresholded at $t>6$. Combined activation maps from both tasks showed similar robust perisylvian language area activation, including inferior frontal gyrus, posterior superior temporal lobe, and fusiform gyrus. Subjects performed well on the rhyming task, which activated left hemisphere cortical regions more selectively than the word generation task. The rhyming task showed less activation than the word generation task in areas typically not considered specifically related to language function, such as the dorsolateral prefrontal cortex and anterior cingulate. The rhyming task is a useful tool for brain mapping and clinical applications, potentially more specific to cortical language areas than verbal fluency. Hum. Brain Mapping 10:99-106, 2000. @ 2000 Wiley-Liss, Inc.
\end{abstract}

Key words: language; phonology; fluency; human; brain; cortex; Broca; Wernicke

\section{INTRODUCTION}

The ability to determine non-invasively the cerebral localization of language functions is important in many clinical settings, such as pre-surgical planning for patients with epilepsy or brain neoplasms. The precise cerebral localization of language sub-functions

*Correspondence to: Joseph T. Lurito, Department of Radiology, Indiana University School of Medicine, 550 N. University Boulevard UH 0615 Indianapolis, IN 46202

E-mail: jlurito@iupui.edu

Received 21 September 1999; Accepted 13 April 2000 in the normal human remains unclear. It is difficult to infer cortical localization of function from clinical lesions, which may disrupt many interrelated functional systems. This problem has become more apparent because of the wealth of data from functional brain imaging studies of language, many of which challenge the traditional models of cortical language organization (Binder, 1997).

Functional magnetic resonance imaging (FMRI) is a relatively new brain activation technique which is useful for mapping many brain areas, such as sensory cortex (Courtney and Ungerleider, 1997; Yetkin et al., 1995b) and various motor areas (Van Oostende et al.,

๑) 2000 Wiley-Liss, Inc. 
1997; Wexler et al., 1997), and association cortex involved in memory (Epstein and Kanwisher, 1998; Fernandez et al., 1998) and language (Binder, 1997; FitzGerald et al., 1997). The major advantage of using FMRI in language mapping is the ability to perform noninvasively a complete battery of tasks designed to probe various language sub-functions in single subjects. This is possible because FMRI uses no ionizing radiation and has high sensitivity. The major disadvantages of FMRI in language mapping are the loud scanner noise, which makes it difficult to present auditory stimuli, and extreme motion sensitivity ( $\mathrm{Wu}$ et al., 1997), which makes overt verbal responses from the subject problematic.

Word generation ("verbal fluency") is a well studied and commonly applied behavioral and FMRI paradigm (Cuenod et al., 1995; Hinke et al., 1993; Stuss et al., 1998). However, data quality and level of brain activation depend on many behavioral factors, including whether the subject's response is spoken or silent (Yetkin et al., 1995a). Although silent paradigms cause significantly less motion artifact (Yetkin et al., 1995a), there is no way to verify performance or correlate behavior with functional activation. We have developed a silent word rhyming paradigm that activates peri-sylvian language areas (Kareken, et al., 2000) and provides behavioral performance data. Direct comparison of brain activation patterns of a single group of subjects performing both the silent fluency and word rhyming tasks should provide important information about the relative efficacy and specificity of dominant hemisphere language activation in these two tasks.

\section{MATERIALS AND METHODS}

Five healthy, right-handed subjects (3 females; mean age 27, standard deviation 2.7 years; education $17.6 \pm 2.7$ years) were recruited and provided written informed consent. The protocol was approved by the local Institutional Review Board. Subjects were instructed in the performance of a novel rhyming task and the fluency task. Both tasks consisted of five 32 second control periods alternated with four 32 second activation periods.

The activation period for the rhyming task consisted of determining whether two visually presented common English words rhymed (Fig. 1A) and then pressing the corresponding button on a response pad using the right hand. To force subvocal articulation and prevent responses based on word appearance, rhyming word pairs $(n=20)$ were orthographically different and non-rhyming pairs $(n=20)$ were orthographically similar to varying degrees. The control period consisted of determining whether two sets of line arrays were identical (Fig. 1B) and responding with the appropriate button press. Ten pairs of words or line arrays were presented during each control or activation period.

The activation periods of the fluency task consisted of sequential visual presentation of two letters of the English alphabet (Fig. 2A). The eight letters were selected and balanced across the four activation periods according to their associative frequencies (Borkowski et al., 1967). Subjects were instructed to silently generate as many words as possible beginning with the presented letter (Cuenod et al., 1995; Yetkin et al., 1995a), with the exception of proper names and different grammatical forms of the same word (e.g. come, coming) (Benton et al., 1994). During the control period, subjects were instructed to visually fixate a meaningless non-letter symbol (Fig. 2B), which was either a character from a foreign language script, such as Arabic, Hebrew, or Mandarin, or an abstract "letterlike" line drawing.

Subjects were observed while practicing each task to ensure proper performance before being placed into the MRI scanner. All visual stimuli were computer generated and presented using an MRI compatible binocular fiberoptic display (Avotec, Inc. Jensen Beach, Florida, USA). The timing of the stimulus presentation was controlled and subject responses were recorded using Stim (Neuroscan, Inc. Sterling, VA, USA).

Subjects were placed in a birdcage head coil and fitted to a bite bar attached to the coil to reduce head motion. All MRI studies were performed on a General Electric (Wisconsin, USA) 1.5 Tesla Signa Echospeed scanner. Anatomic scans, including a heavily T1weighted 3D spoiled grass sequence, were obtained prior to functional images. Whole brain gradient echo echoplanar FMRI was performed with the following imaging parameters: 15 coronal slices of thickness $7 \mathrm{~mm}$ with inter-slice gap of $2 \mathrm{~mm}$; TR $2 \mathrm{~s}$, TE $50 \mathrm{~ms} ; 90^{\circ}$ flip angle; $64 \times 64$ matrix; bandwidth $125 \mathrm{kHz}$; and field of view $24 \mathrm{~cm}$. Each set of 15 images was collected every 2 seconds during both the control and activation states for the rhyming and fluency tasks. The locations of the coronal functional scans were chosen to cover the entire peri-sylvian region and as much of the frontal and occipital lobes as possible. The order of performance of the rhyming and fluency tasks was randomized across subjects.

The raw image data were Hamming filtered (Lowe and Sorenson, 1997) to improve signal to noise ratio with minimal reduction in spatial resolution. A leastsquares fit of each single pixel time series to a boxcar function, which represents the idealized activation 


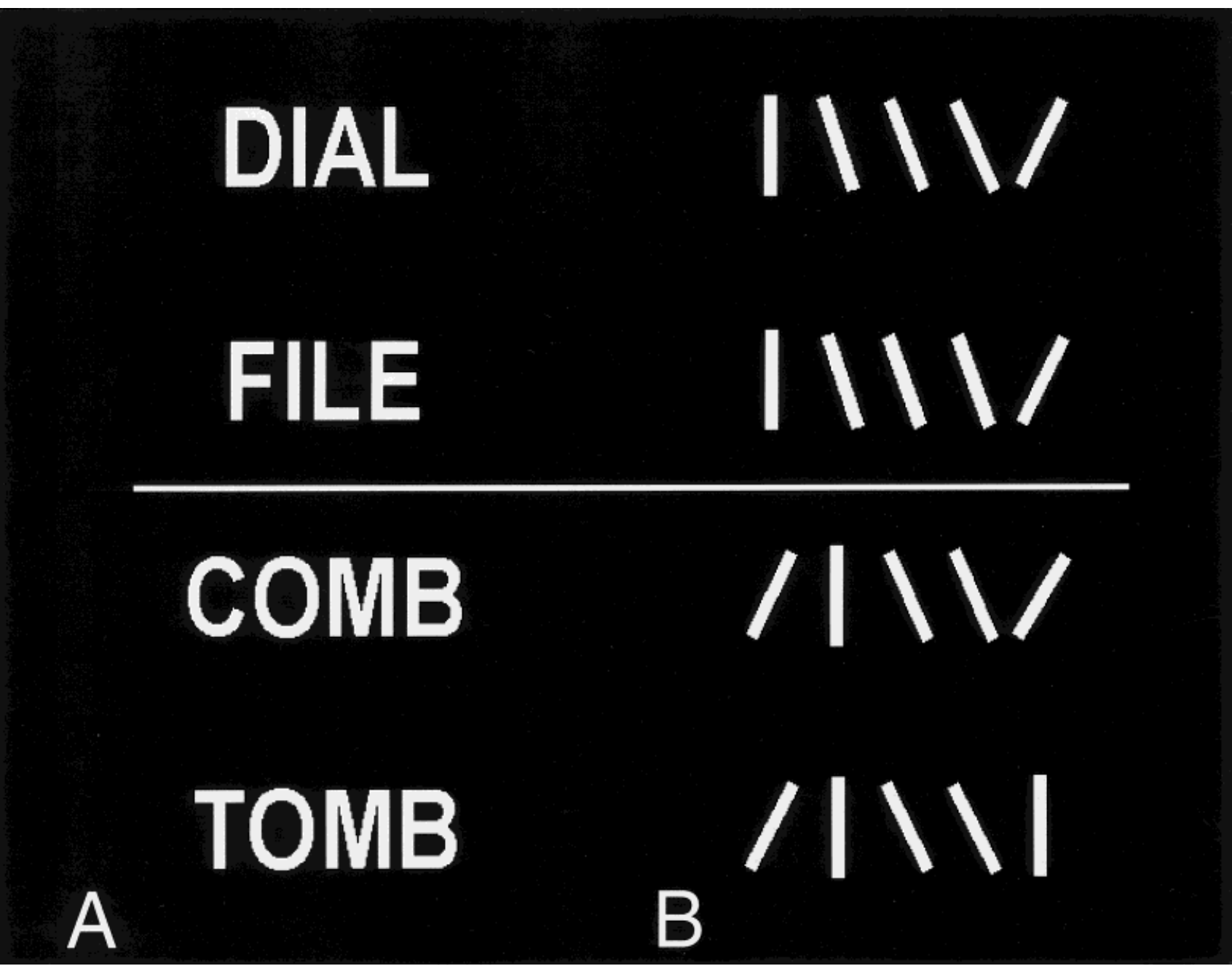

Figure I.

A. The word pairs may look different, but still rhyme (top left), or look similar, but not rhyme (bottom left). Orthographically similar word pairs which rhyme and orthographically different word pairs which do not rhyme were also presented. B. The stick pairs which were not identical were usually quite similar (bottom right).

paradigm, was performed using three free parameters and a baseline slope and intercept (Lowe and Russell, 1999). The fit amplitude divided by the error gives a $\mathrm{t}$-statistic for each pixel. These activation maps were thresholded at $\mathrm{t}>3.5$ and superimposed on coronal T1-weighted anatomic images.

These single subject activation maps were combined as follows. The maps were interpolated to $256^{3}$ and then transformed to the true coronal (Talairach and Tournoux, 1988) plane. The five maps were summed pixel by pixel and the combined map was thresholded at $t>6$ (corresponding to a single pixel confidence level of roughly $10^{-27}$ ) and displayed on T1-weighted anatomic scans from one of the subjects, also transformed into the Talairach coronal plane. The locations of regions of activation were determined from the Talairach coordinates.

\section{RESULTS}

The fluency task resulted in significant activation for combined subject data in several left cerebral hemisphere areas (Table I; Fig. 3, middle column), including: a confluent region of activated foci extending from the motor cortex to the junction of areas $9 / 46$ (these are not listed separately in Table I because of inability to separate reliably into individual peaks); Broca's area; a band from deep to the posterior superior temporal gyrus to the posterior middle temporal gyrus; supramarginal gyrus; posterior lateral fusiform 


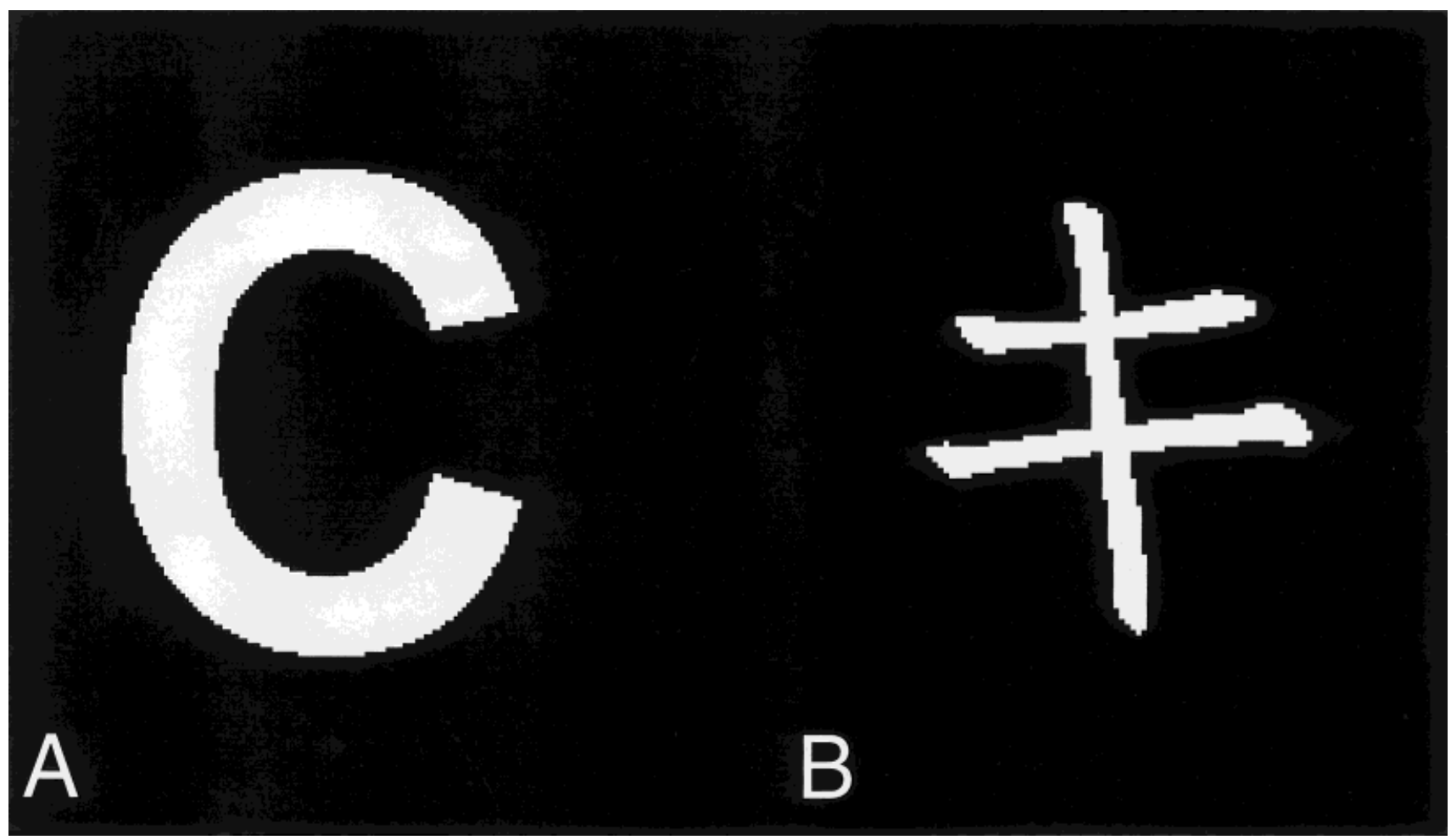

Figure 2.

Examples of stimuli used for the fluency task. English letter (A). Foreign language character (B), which is "letter-like" in appearance, but meaningless to the subjects.

gyrus; a band from the supplementary motor area to the anterior cingulate cortex; a band from the upper to lower inferior parietal lobule; and caudate nucleus. Few right hemisphere areas were activated: the middle frontal gyrus; inferior frontal gyrus bordering the anterior insula; deep to area 22 in the posterior superior temporal gyrus. Activation was also noted in the superior and inferior cerebellar cortex, right more than left.

Subjects performed accurately on both the activation $(87.5 \pm 5.9 \%$ correct $)$ and control $(90.0 \pm 8.9 \%$ correct) portions of the rhyming task. The rhyming task (Table I; Fig. 3, left column) combined subject data showed activation in the left dorsolateral prefrontal cortex (BA 9/46) less in spatial extent and lower in statistical significance as compared to the fluency task. Activation in Broca's area and the posterior lateral fusiform gyrus was very similar in location and significance to the fluency task. The activation in the supramarginal gyrus was more significant than in the fluency task. The activation in the posterior superior temporal gyrus was much less significant. A band of activation deep to and within the superior bank of the left middle temporal gyrus was noted. The right hemisphere showed only minimal activation in the homo- logue to Broca's area. There was no activation in the left inferior parietal lobule outside the supramarginal gyrus, caudate, anterior cingulate, SMA or premotor cortex, the right DLPFC, or on either side of the cerebellum.

Single subject activation maps were similar to the combined subject maps as shown in Figure 4.

\section{DISCUSSION}

There are many similarities between the regions activated here and those of other FMRI studies employing silent verbal fluency. Most studies have shown activation in the major peri-sylvian language regions, including Broca's area (Chee et al., 1999; Cuenod et al., 1995; Hinke et al., 1993; Ojemann et al., 1998; Sadato et al., 1998) and posterior temporal and parietal opercular foci (Chee et al., 1999; Cuenod et al., 1995; Sadato et al., 1998). Activation in other regions is more variable. For example, the posterior fusiform gyrus, was seen in most, (Chee et al., 1999; Cuenod et al., 1995; Sadato et al., 1998), but not all studies (Ojemann et al., 1998) and similar variability was noted for the anterior cingulate, supplementary motor area, and the dorsolateral prefrontal cortex. 
TABLE I. Centroids and peak $t$ score of activated regions

\begin{tabular}{|c|c|c|c|c|}
\hline \multirow{2}{*}{$\begin{array}{l}\text { Talairach } \\
\text { coordinates }\end{array}$} & \multirow[b]{2}{*}{ Brain region } & \multirow{2}{*}{$\begin{array}{l}\text { Brodmann } \\
\text { area }\end{array}$} & \multicolumn{2}{|c|}{ Peak $t$} \\
\hline & & & Rhyming & Fluency \\
\hline \multicolumn{5}{|l|}{ Left } \\
\hline$(-58,-54,6)$ & MTG & 21 & & 25.7 \\
\hline$(-56,-32,8)$ & STG & 22 (Wernicke) & & 20.3 \\
\hline$(-46,-51,-19)$ & Fusiform gyrus & 37 & & 21.0 \\
\hline$(-43,-61,45)$ & IPL near IPS & 40 (near 7) & & 21.8 \\
\hline$(-33,-60,38)$ & IPL deep in IPS & 40 & & 18.2 \\
\hline$(-47,-48,42)$ & IPL & 40 & & 19.5 \\
\hline$(-5,9,46)$ & Anterior cingulate in sulcus & 32 & & 38.5 \\
\hline$(-5,-8,58)$ & SMA & 6 & & 32.8 \\
\hline$(-53,14,6)$ & IFG & 44 (Broca) & & 30.6 \\
\hline$(-27,-57,-24)$ & Superior cerebellum & & & 14.9 \\
\hline$(-46,32,25)$ & MFG & 9, 46 (DLPFC) & 20.2 & \\
\hline$(-49,11,32)$ & Junction MFG, IFG & 8,9 & 21.0 & \\
\hline$(-55,13,7)$ & IFG & 44 (Broca) & 23.5 & \\
\hline$(-63,-31,26)$ & Supramarginal gyrus & 40 & 30.0 & \\
\hline$(-57,-46,1)$ & MTG in bank STS & 21 & 16.1 & \\
\hline$(-44,-59,-18)$ & Fusiform gyrus & 37 & 18.9 & \\
\hline \multicolumn{5}{|l|}{ Right } \\
\hline$(46,-47,17)$ & STG near STS & 22 & & 23.6 \\
\hline$(40,21,11)$ & Junction IFG, insula & $44 / 45$ & & 15.7 \\
\hline$(35,32,41)$ & MFG & 8 & & 17.3 \\
\hline$(33,-59,-28)$ & Superior cerebellum & & & 24.4 \\
\hline$(28,-60,-54)$ & Inferior cerebellum & & & 23.6 \\
\hline$(55,7,6)$ & IFG & 44 & 12.6 & \\
\hline
\end{tabular}

Abbreviations for Table: IFG, MFG = inferior, middle frontal gyrus; MTG, STG = middle, superior temporal gyrus; IPL = inferior parietal lobule; IPS = intraparietal sulcus; STS = superior temporal sulcus; $\mathrm{SMA}=$ supplementary motor area; DLPFC $=$ dorsolateral prefrontal cortex.

Both rhyming and fluency give excellent peri-sylvian language area activation. The differences observed here between regional activation in the rhyming task and fluency task indicate that the rhyming task has more "specific" activation of language regions. The SMA and anterior cingulate are activated in a variety of tasks, many of which have no apparent language component, and are generally not considered language regions. The rhyming task may not show activation in these regions because, unlike the fluency task, both activation and control periods have similar stimulus characteristics and response demands. Both require similar visual scanning of the stimuli, sustained attention, and response selection. Impaired verbal fluency also often reflects frontal lobe impairment in the absence of aphasia or other language abnormalities (Baldo and Shimamura, 1998; Stuss et al., 1998). As such, it is commonly interpreted as a measure of "executive function" requiring internally generated response initiation, mental flexibility, and a relatively unconstrained strategic search of the mental lexicon. A greater expanse of dorsolateral prefrontal cortex may therefore be apparent in the fluency task because of the executive demands of the activation task. However, more importantly, it is likely that the lack of a matched control task requiring executive function that leads to the relative nonspecificity of the fluency task, which also evoked more right hemisphere activation than the rhyming task. Although left dorsolateral prefrontal cortex damage clearly impairs verbal fluency, right superior medial damage also impairs fluency (Stuss et al., 1998). Thus, the right frontal lobe likely participates in this task as well.

The rhyming task has several advantages over the fluency task. Behavioral performance is easily quantified, allowing for more confident interpretation of the activation maps. The activation noted in the rhyming task is more restricted, but not completely so, to cortical regions generally considered language areas. There is stronger hemispheric lateralization, which could prove useful in clinical applications, such as for 

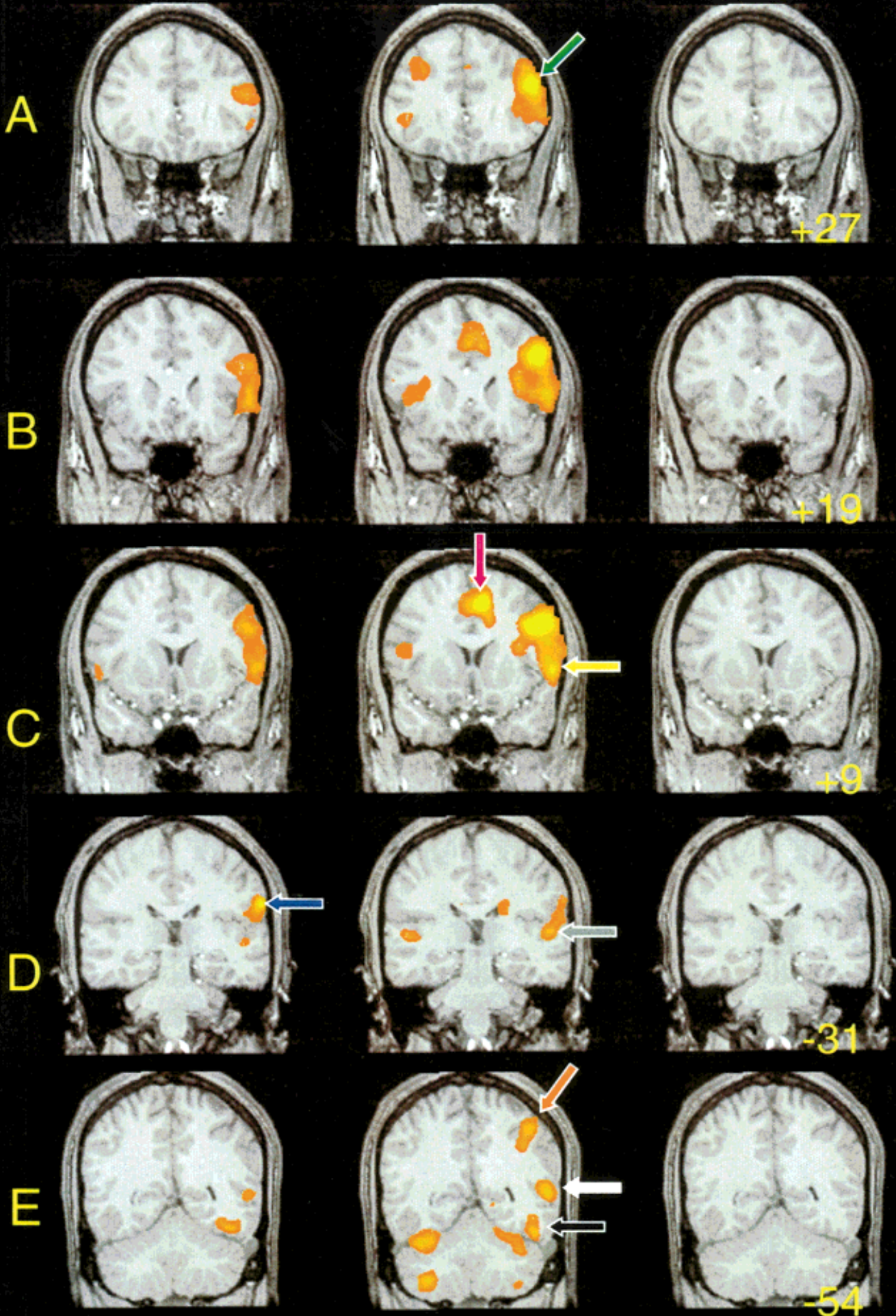

\section{Rhyming Fluency Anatomy}

Figure 3.

Activation Student $\mathrm{t}$ maps for the rhyming (left) and fluency (middle) tasks superimposed on coronal TI-weighted anatomic images (right) from anterior (A) to posterior (E). The images are displayed in radiographic convention (left hemisphere on right of image) and the Talairach antero-posterior (y) coordinates of the coronal sections are given. Activated regions include: bilateral dorsolateral prefrontal cortex (green arrow); Broca's area (yellow arrow); anterior cingulate cortex (red arrow); left posterior area 22 (white arrow); left supramarginal gyrus (blue arrow); cortex deep to posterior area 2l/22 (grey arrow); left posterior fusiform gyrus (black arrow); left inferior parietal lobule (orange arrows, area 39); bilateral superior and inferior cerebellar lobules; and left caudate. 

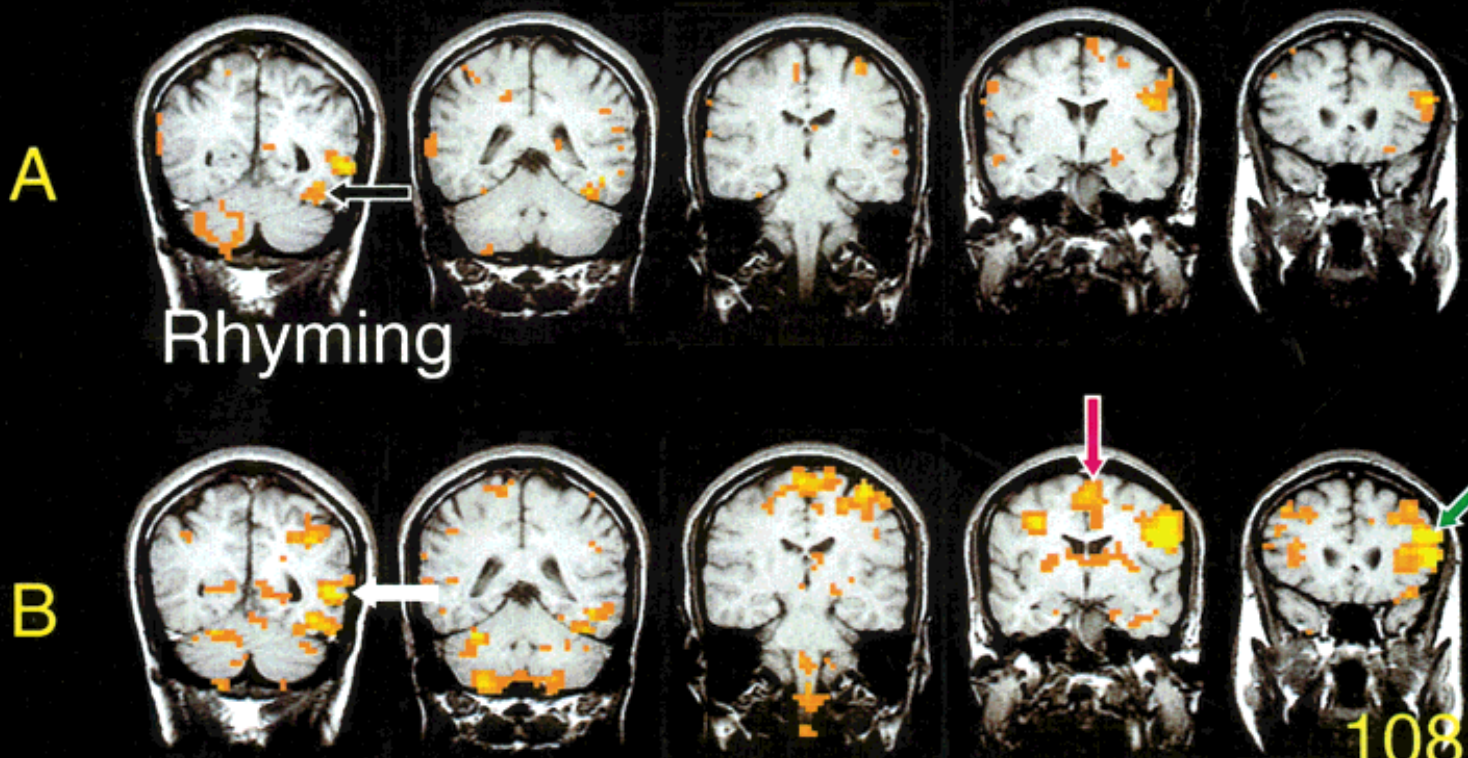

\section{Fluency}
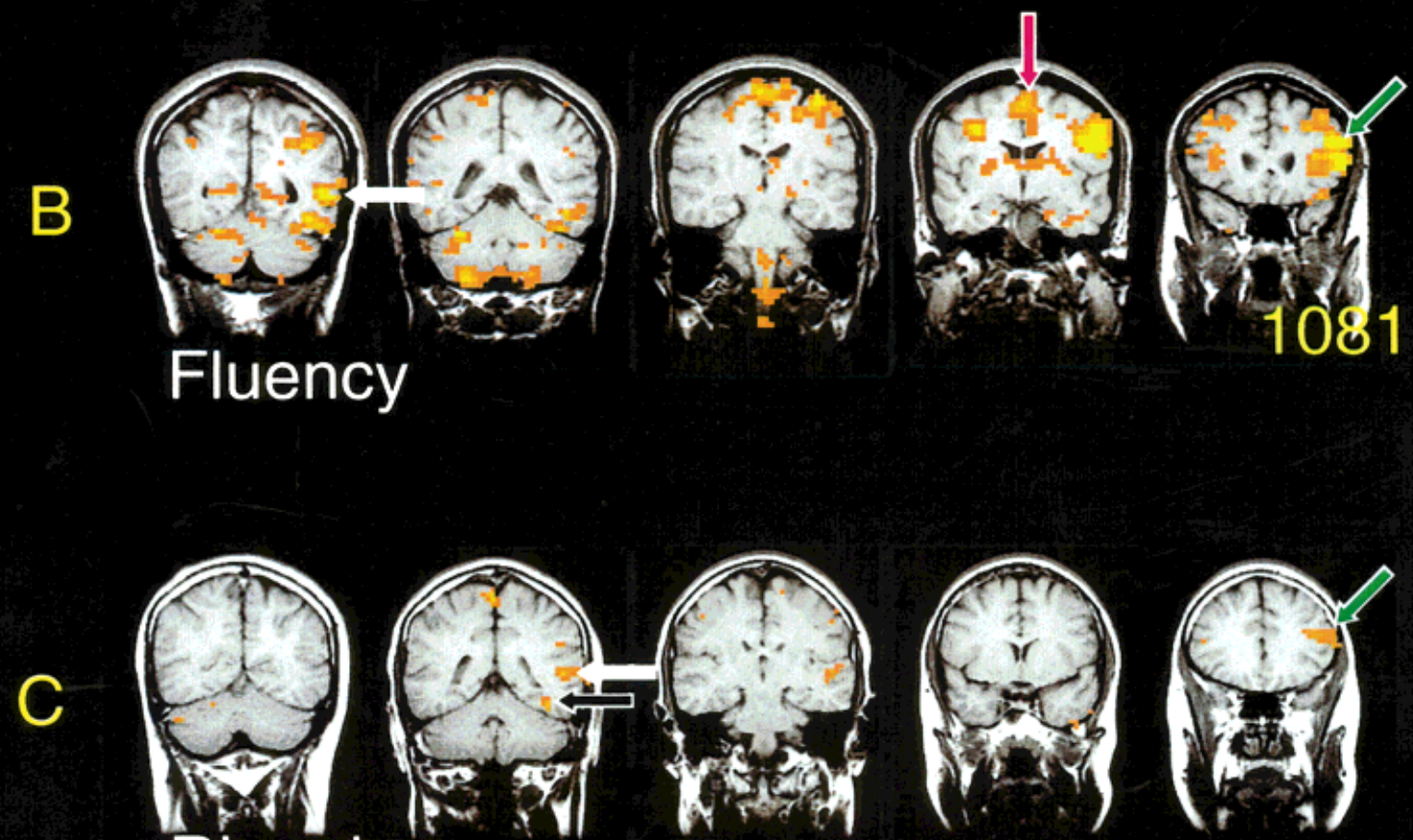

\section{Rhyming}

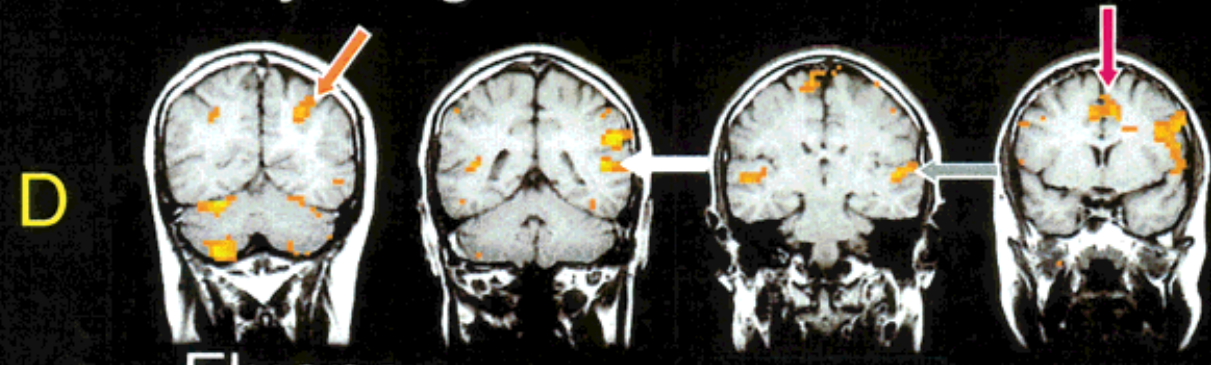

Fluency

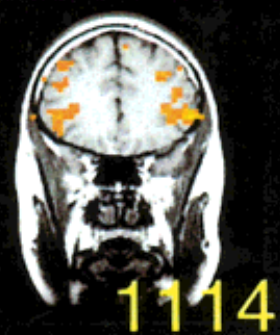

Figure 4.

Activation Student $t$ maps (threshold $t>3.5$, single pixel confidence level approximately $10^{-4}$ ) for two individual subjects superimposed on five coronal anatomic sections arranged from posterior to anterior reading left to right. Maps from both the rhyming $(\mathbf{A}, \mathbf{C})$ and fluency $(\mathbf{B}, \mathbf{D})$ tasks are similar to the combined activation maps (see Fig. 3). The images are displayed in radiographic convention and colored arrows label the same structures as in Figure 3. 
a non-invasive alternative to the Wada test for hemispheric dominance.

\section{CONCLUSION}

The rhyming task is useful for using FMRI to localize cerebral language regions. Future research on clinical patients and in subjects with atypical language dominance are required to confirm its usefulness for both brain mapping and clinical applications.

\section{ACKNOWLEDGMENTS}

The authors wish to thank Julie Sims, BSRT for assistance in performing the MRI examinations and Rachel Lindop for manuscript preparation.

\section{REFERENCES}

Baldo JV, Shimamura AP. (1998): Letter and category fluency in patients with frontal lobe lesions. Neuropsychology 12:259-67.

Benton A, Hamsher K, Sivan A. (1994): Multilingual Aphasia Examination. New York: Oxford University Press.

Binder JR. (1997): Neuroanatomy of language processing studied with functional MRI. Clin Neurosci 4:87-94.

Borkowski J, Benton A, Spreen O. (1967): Word fluency and brain damage. Neuropsychologia 5:135-140.

Chee MW, Tan EW, Thiel T. (1999): Mandarin and English single word processing studied with functional magnetic resonance imaging. J Neurosci 19:3050-6.

Courtney SM, Ungerleider LG. (1997): What fMRI has taught us about human vision. Curr Opin Neurobiol 7:554-61.

Cuenod CA, Bookheimer SY, Hertz-Pannier L, Zeffiro TA, Theodore WH, Le Bihan D. (1995): Functional MRI during word generation, using conventional equipment: a potential tool for language localization in the clinical environment. Neurology 45:1821-7.

Epstein R, Kanwisher N. (1998): A cortical representation of the local visual environment. Nature 392:598-601.

Fernandez G, Weyerts H, Schrader-Bolsche M, Tendolkar I, Smid HG, Tempelmann C, Hinrichs H, Scheich H, Elger CE, Mangun GR, Heinze HJ. (1998): Successful verbal encoding into episodic memory engages the posterior hippocampus: a parametrically analyzed functional magnetic resonance imaging study. J Neurosci 18:1841-7.

FitzGerald DB, Cosgrove GR, Ronner S, Jiang H, Buchbinder BR, Belliveau JW, Rosen BR, Benson RR. (1997): Location of lan- guage in the cortex: a comparison between functional MR imaging and electrocortical stimulation. AJNR Am J Neuroradiol 18:1529-39.

Hinke RM, Hu X, Stillman AE, Kim SG, Merkle H, Salmi R, Ugurbil K. (1993): Functional magnetic resonance imaging of Broca's area during internal speech. Neuroreport 4:675-8.

Kareken DA, Lowe MJ, Lurito JT, Chen SH, Mathews VP. (2000): Functional neuroanatomy of Word Rhyming During Functional Magnetic Resonance Imaging. Neuropsych Neuropsychol Bahav Neurol (in press).

Lowe MJ, Russell DP. (1999): Treatment of baseline drifts in fMRI time series analysis. J Comput Assist Tomogr 23:463-73.

Lowe MJ, Sorenson JA. (1997): Spatially filtering functional magnetic resonance imaging data. Magn Reson Med 37:723-9.

Ojemann JG, Buckner RL, Akbudak E, Snyder AZ, Ollinger JM, McKinstry RC, Rosen BR, Petersen SE, Raichle ME, Conturo TE. (1998): Functional MRI studies of word-stem completion: reliability across laboratories and comparison to blood flow imaging with PET. Hum Brain Mapp 6:203-15.

Sadato N, Yonekura Y, Yamada H, Nakamura S, Waki A, Ishii Y. (1998): Activation patterns of covert word generation detected by fMRI: comparison with 3D PET. J Comput Assist Tomogr 22:945-52.

Stuss DT, Alexander MP, Hamer L, Palumbo C, Dempster R, Binns M, Levine B, Izukawa D. (1998): The effects of focal anterior and posterior brain lesions on verbal fluency. J Int Neuropsychol Soc 4:265-78.

Talairach J, Tournoux P. (1988): Co-planar stereotaxic atlas of the human brain: An approach to medical cerebral imaging. Stuttgart; New York: G. Thieme

Van Oostende S, Van Hecke P, Sunaert S, Nuttin B, Marchal G. (1997): FMRI studies of the supplementary motor area and the premotor cortex. Neuroimage 6:181-90.

Wexler BE, Fulbright RK, Lacadie CM, Skudlarski P, Kelz MB, Constable RT, Gore JC. (1997): An fMRI study of the human cortical motor system response to increasing functional demands. Magn Reson Imaging 15:385-96.

Wu DH, Lewin JS, Duerk JL. (1997): Inadequacy of motion correction algorithms in functional MRI: role of susceptibility-induced artifacts. J Magn Reson Imaging 7:365-70.

Yetkin FZ, Hammeke TA, Swanson SJ, Morris GL, Mueller WM, McAuliffe TL, Haughton VM. (1995a): A comparison of functional MR activation patterns during silent and audible language tasks. AJNR Am J Neuroradiol 16:1087-92.

Yetkin FZ, Papke RA, Mark LP, Daniels DL, Mueller WM, Haughton VM. (1995b): Location of the sensorimotor cortex: functional and conventional MR compared. AJNR Am J Neuroradiol 16: 2109-13. 